\title{
Fuel Efficient Computation in Passive Self-Assembly
}

\author{
Robert Schweller* $\quad$ Michael Sherman ${ }^{\dagger}$
}

\begin{abstract}
In this paper we show that passive self-assembly in the context of the tile self-assembly model is capable of performing fuel efficient, universal computation. The tile self-assembly model is a premiere model of self-assembly in which particles are modeled by four-sided squares with glue types assigned to each tile edge. The assembly process is driven by positive and negative force interactions between glue types, allowing for tile assemblies floating in the plane to combine and break apart over time. We refer to this type of assembly model as passive in that the constituent parts remain unchanged throughout the assembly process regardless of their interactions. A computationally universal system is said to be fuel efficient if the number of tiles used up per computation step is bounded by a constant. Work within this model has shown how fuel guzzling tile systems can perform universal computation with only positive strength glue interactions 32 . Recent work has introduced space-efficient, fuel-guzzling universal computation with the addition of negative glue interactions and the use of a powerful non-diagonal class of glue interactions 19. Other recent work has shown how to achieve fuel efficient computation 27] within active tile self-assembly. In this paper we utilize negative interactions in the tile self-assembly model to achieve the first computationally universal passive tile self-assembly system that is both space and fuel-efficient. In addition, we achieve this result using a limited diagonal class of glue interactions.
\end{abstract}

\footnotetext{
${ }^{*}$ Department of Computer Science, University of Texas - Pan American, rtschweller@utpa.edu This author's research was supported in part by National Science Foundation Grant CCF-1117672.

$\dagger$ Department of Computer Science, University of Texas - Pan American, mjsherman@utpa.edu This author's research was supported in part by National Science Foundation Grant CCF-1117672.
} 


\section{Introduction}

Self-assembly is the process by which systems of simple objects organize themselves through local interactions into larger, more complex objects. There are perhaps two categories of self-assembly: passive and active. In passive self-assembly the objects of the system are simple, stagnant particles that interact simply by surface chemistry and geometry. In contrast, objects within an active self-assembly model may be permitted to move, rotate, adjust state, or add and remove bonding domains based on local interactions. Put another way, active self-assembly extends traditional passive self-assembly by considering the objects of the system to be simple robots with abilities that vary according to the particular model considered.

The study of passive self-assembly is important for multiple reasons. First, many theoretical models of active self-assembly do not currently have satisfactory implementations at the nanoscale. In contrast, many computationally interesting passive tile self-assembly constructions are seeing experimental success based on DNA implementations 7, 23, 29. Further, it is plausible that many active self-assembly models will see implementation through constructions built up within passive self-assembly models. For example, the system proposed in this paper which implements a space-efficient, fuel efficient universal computation system might be modified to serve as the internal machinery for a larger, active component, thus potentially allowing for a passive self-assembly system to simulate the behavior of a more powerful active system. Finally, it is important to understand the power and limits of passive self-assembly to better understand when active components are truly necessary.

In this paper we focus on an established model of passive self-assembly, the tile assembly model (TAM). Monomers in the TAM are unit squares with glue types assigned to edges. Self-assembly is driven by a large (infinite) number of copies of a set of tile types floating about, bumping into one another in the plane, and potentially sticking together when glue affinities exceed some set threshold. While simple, the TAM has been extensively studied [1, 6, $8,18,21,22,24,26,30,31]$ and shown to be capable of universal computation [32], and even recently shown to be intrinsically universal 20 in the case of the abstract TAM.

Within the TAM, we consider the problem of improving existing universal computation results by obtaining fuel-efficiency. A TAM system that simulates a Turing machine is said to be fuel-efficient if only a constant number of tile types are used up per computation step. This requirement, or close approximations to it, are of fundamental importance for the implementation of scalable molecular computers. Unfortunately, all passive variants of the TAM have failed to yield fuel-efficient universal computation.

To address this fuel-deficiency, we consider the TAM with the added power of negative glue interactions. The power of negative force interactions stems from the possibility of stable assemblies coming together with strong affinities and yet producing unstable products that subsequently break apart into pieces that are different from the original pair of assemblies. By careful engineering, this property can be harnessed to allow assemblies to attach to specific locations of large assemblies, surgically pry off and replace small portions of the assembly, and finally detach the replacement mechanism and repeat the process. If you imagine the large assembly representing the tape of a Turing machine and the location of attachment is at the current head location, you get a high level overview of our Turing machine simulation that only uses up a fixed number of tile types per computation step. This stands in contrast to previous fuel guzzling work in which each computation step is simulated by the assembly of a complete, slightly modified, copy of the entire tape.

\subsection{Related Work}

Doty et. al. [19] first considered the effect of negative interactions in self-assembly and showed how to design space efficient computational self-assembly systems by utilizing negative glues to slice off a previous assembly state after computing the next state. This state transition construction can be applied to the simulation of a Turing machine. However, their construction does not achieve fuel efficiency in that each computational step uses up a number of tiles on the order of the current size of the tape. Their result is also not applicable to our graph traversal system in that their technique, modified to the graph traversal problem, would lead to dead end assemblies that violate the required dynamics of the graph traversal. Finally, their paper utilizes a very powerful non-diagonal version of the tile self-assembly glue function. We restrict ourselves to the much more restricted diagonal class of glue functions which is likely more feasible for experimental implementation. 
Additionally, Doty et. al. provide an elegant amortized analysis proof showing a limit to the extent to which tile reuse can be achieved with negative force interactions within the TAM. We implicitly use their result in that it allows us to reasonably simplify our definition of fuel usage to not deal with tile reuse.

Some recent work has been done that shows provable increases in power of active self-assembly systems over passive TAM systems. Recent work by Woods et. al. 33 has shown that local rule based active selfassembly systems are able to assemble large shapes in poly-logarithmic time when the monomers of the system are able to perform operations such as pushing a row of placed monomers a unit of distance in some direction. Another recent work by Padilla et. al. 27 considers an active tile self-assembly model motivated by a DNA strand replacement mechanism in which tiles can pass simple, fire-once signals from one tile edge to another. They show that this simple mechanism allows for the implementation of fuel-efficient universal computation, as well as additional efficiencies that are provably impossible within the passive TAM.

Additional investigations have looked into self-assembly models that allow self-assembly detachment, a key requirement for the implementation of space efficient computations [1,6,24, 31. Other recent work has considered negative glues within the TAM to achieve universal computation with a very limited set of glues at temperature-1 28.

Paper layout. In Section 2 we define the tile assembly model, as well as present definitions that define our graph traversal and Turing machine simulation problems. In Section 3 we present our construction for fuel efficient graph walking. Finally, in Section 5 , we extend our graph walking construction to simulate Turing machines.

\section{Definitions and Model}
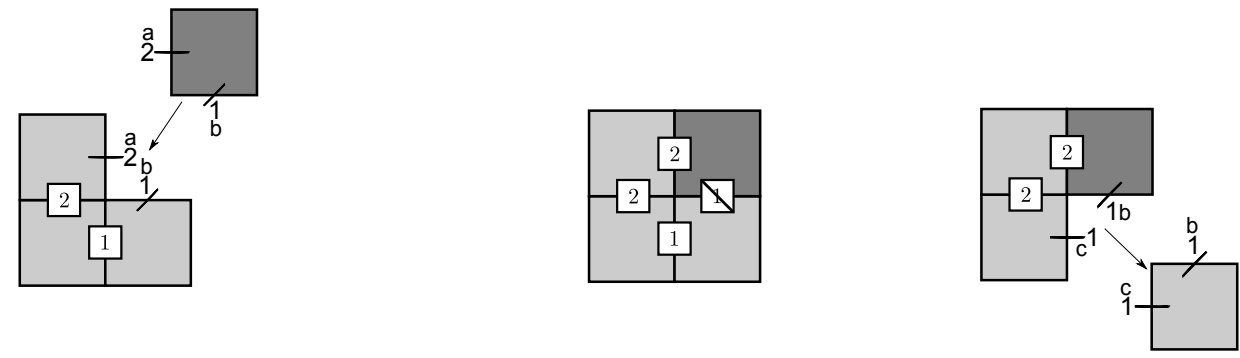

Figure 1: This figure introduces our notation for our constructions, as well as a simple example of combination and detachment events. We denote positive glues with vertical and horizontal lines protruding from tile edges along with numbers and labels denoting glue strength and type. Negative strength glues are labeled with slanted lines. Finally, bonded glues between adjacent tiles are depicted with a number denoting the bond strength inscribed within a box between the bonded tile edges. (a) The 3-tile assembly on the left and the singleton gray tile are combinable into a $2 \times 2$ square shown in (b) as the cut between the 2 assemblies yields strength 1 . Note, however, that the producible $2 \times 2$ square in (b) is not stable and is breakable into the assemblies shown in (c).

In this section we first define the two-handed tile self-assembly model with both negative and positive strength glue types and diagonal glue functions. We also formulate the problem of designing a tile assembly system that walks a given input graph, as well as the concept of fuel efficiency.

\subsection{Tile Self-Assembly Model}

Tiles. Consider some alphabet of glue types $\Pi$. A tile is a finite edge polygon with some finite subset of border points each assigned some glue type from $\Pi$. Further, each glue type $g \in \Pi$ has some rational number 
strength $\operatorname{str}(g)$. Finally, each tile may be assigned a finite length string label. In this paper we consider a special class of tiles that are unit squares of the same orientation with at most one glue type per face, with each glue being placed exactly in the center of the tile's face.

Assemblies. An assembly is a set of tiles whose interiors do not overlap. For a given assembly $\Upsilon$, define the bond graph $G_{\Upsilon}$ to be the weighted graph in which each element of $\Upsilon$ is a vertex, and each edge weight between tiles is the sum of the strengths of the overlapping, matching glue points of the two tiles. An assembly $C$ is said to be stable if the bond graph $G_{C}$ has min-cut at least 1, and unstable if the min-cut is less than 1. Note that if the set of border points of all tiles in an assembly is not a connected set, then the assembly cannot be stable. Note that only overlapping glues that are the same type contribute a non-zero weight, whereas overlapping, non-equal glues always contribute zero weight to the bond graph. The property that only equal glue types interact with each other is referred to as the diagonal glue function property and is perhaps more feasible for experimental implementation than more general glue functions. Additionally, with the square tiles considered in this paper, stable assemblies will necessarily consist of tiles stacked face to face, forming a subset of the $2 \mathrm{D}$ grid.

For an assembly $A$, let $A^{*}$ denote the set of all assemblies that are equal to $A$ up to translation. For a set of assemblies $T$, let $T^{*}$ denote the set of all assembly sets $A^{*}$ such that $A \in T$. Put another way, $T^{*}$ is a set that represents a set of assemblies $T$ if we do not care about translation.

Breakable Assemblies. For an (unstable) assembly $C$ whose bond graph $G_{C}$ has a cut into assemblies $A$ and $B$ with weight less than 1 , we say that $C$ is breakable into $A$ and $B$.

Combinable Assemblies. Informally, two assemblies $A$ and $B$ are said to be combinable into an assembly $C$ if the assemblies can be translated together in such a way that they do not overlap and the sum of the matched glue strengths between the two assemblies is at least 1. Formally, consider two assemblies $A$ and $B$. If $B$ can be translated into $B^{\prime}$ such that $C=A \cup B^{\prime}$ is a valid (not overlapping) assembly such that the cut of $G_{A \cup B^{\prime}}$ into $A$ and $B^{\prime}$ has strength at least 1 , then we say that $A$ and $B$ are combinable into $C$.

Note that $A$ and $B$ may be combinable into an assembly that is not stable. This is a key property that is leveraged throughout our constructions. See Figure 1 for an example.

Producible Assemblies. A set of initial assemblies $T$ has an associated set of producible assemblies, $\operatorname{PROD}_{T}$, which define what assemblies can grow from the initial set $T$ by any sequence of combination and break events. Formally, $T \subseteq \operatorname{PROD}_{T}$ as a base case set of producible assemblies. Further, given any $A, B \in \mathrm{PROD}_{T}$

with $A$ and $B$ combinable into $C$, then $C \in \operatorname{PROD}_{T}$, and for any $C \in \operatorname{PROD}_{T}$ where $C$ is breakable into $A$ and $B$, then $A, B \in \mathrm{PROD}_{T}$. Put another way, the set of producible assemblies contains any assembly that can be obtained by a valid sequence of combinations and breaks, starting from the initial set of assemblies $T$. We further define the set of terminal assemblies, TERM ${ }_{T}$, to be the subset of PROD $_{T}$ such that no element of $\mathrm{TERM}_{T}$ can undergo a break or combination transition with another element of $\mathrm{PROD}_{T}$. Note that $\mathrm{PROD}_{T}=$ $\bigcup_{X \in \operatorname{PROD}_{T}^{*}} X$ and $\operatorname{TERM}_{T}=\bigcup_{X \in \operatorname{TERM}_{T}^{*}} X$.

Typically, we require that an initial assembly set consist only of stable assemblies. When $T$ consists only of assemblies that are singleton tiles, $T$ is called a tile set.

\subsection{Additional Definitions}

Valid Assembly Sequence. For an initial assembly set $T$, a valid assembly sequence for $T$ is any sequence of assemblies $S=\left\langle a_{1}, \ldots a_{k}\right\rangle$ such that each $a_{i} \in \operatorname{PROD}_{T}$, and for each $i$ from 1 to $k-1, a_{i}$ is either combinable with some $b \in \operatorname{PROD}_{T}$ to form $a_{i+1}$, or $a_{i}$ is breakable into $a_{i+1}$ and $b$ for some assembly $b$. In addition, a valid assembly sequence $S$ is said to be $\ell$-focused for label $\ell$ if each assembly in $S$ contains at least one tile with label $\ell$. A valid assembly sequence is said to be nascent if $a_{1} \in T$. 
For a given partial function $f: \operatorname{PROD}_{T} \rightarrow V$ for a set $V$, define function $f^{\prime}\left(\left\langle a_{1}, \ldots a_{k}\right\rangle\right)$ to be a function from assembly sequences over $\mathrm{PROD}_{T}$ to sequences over $V$ defined by replacing each $a_{i}$ with $f\left(a_{i}\right)$ if $f\left(a_{i}\right)$ is defined, and deleting $a_{i}$ if $f\left(a_{i}\right)$ is not defined.

Graph Walking Assemblies. Consider a directed graph $G=(V, E)$, a start vertex $s \in V$, and an initial assembly set $T$ in which some tiles of some assemblies are labeled with $\ell$. $T$ is said to walk graph $G$ if there exists a partial function $f: \operatorname{PROD}_{T} \rightarrow V$ such that:

1. (assembly sequences are walks) For any $\ell$-focused nascent assembly sequence $S$ of $T, f^{\prime}(S)$ is a valid walk of $G$ starting at vertex $s$.

2. (walks are assembly sequences) For any walk $W$ of $G$ starting at vertex $s$, there exists a nascent, $\ell$-focused assembly sequence $S$ such that $f^{\prime}(S)=W$.

3. (no undesired dead-ends) Consider an edge $(u, v) \in E$. Then for any $\ell$-focused assembly sequence $S$ such that $f^{\prime}(S)=\langle u\rangle$, there exists an $\ell$-focused assembly sequence $R$ such that $S$ is a prefix of $R$ and $f^{\prime}(R)=\langle u, v\rangle$.

In this definition, the $\ell$ label is meant to denote tiles and assemblies that are meant to be part of the output, and not garbage. That is, to walk a graph efficiently there will necessarily be dead-end garbage assemblies. Our definition thus requires that all such garbage assemblies be free of the $\ell$ label and thus can be distinguished from the assemblies that represent the graph walking assemblies. In a sense, our definition states that if you stare at one $\ell$-labeled tile within an assembly, over time the assembly containing that tile will be guaranteed to walk the graph correctly, whereas if you stare at a portion of the assembly that is not labeled with the $\ell$, it is possible that portion may detach and become garbage at some point.

Fuel Efficiency. Consider two assemblies $a$ and $b$. Define $f u e l(a, b)$ to be $|b|-|a|$ if $|b| \geq|a|$, and zero otherwise. For an assembly sequence $A=\left\langle a_{1}, \ldots a_{k}\right\rangle$, define $\operatorname{fuel}(A)$ to be $\sum \operatorname{fuel}\left(a_{i}, a_{i+1}\right)$.

For a tile set $T$ that walks a directed graph $G, T$ is said to be fuel efficient if for all $\ell$-focused, nascent assembly sequences $S$, fuel $(S) /\left|f^{\prime}(S)\right|=O(1)$. That is, for all possible walks, the average fuel per vertex transition is bounded by a constant.

\section{Graph Walking}

In this section we construct a tile assembly set for the fuel-efficient walk of a given input directed graph:

Theorem 3.1. For any given finite directed graph $G=(V, E)$, there exists a fuel efficient initial assembly set $T$ that walks $G$. Further, $T$ contains at most $O(|V|+|E|)$ distinct assemblies each of $O(1)$ size.

This theorem can be slightly strengthened in that it is possible to modify the construction so that $T$ consists of only singleton tiles. However, for clarity we do not present this modification.

The construction for this result serves two purposes. First, we believe the result itself is interesting. One example corollary is the ability to implement oscillator self-assembly systems with an example given in Section 4. The second purpose is that this construction presents some of the key techniques in a simplified form that we will extend to construct our main result: fuel efficient Turing machines.

The remainder of this section describes the construction for the proof of this theorem.

\subsection{Template Assembly Set}

The following tile assembly template allows one to create an assembly set that can non-deterministically walk across a directed graph. This template set creates an assembly set that has $O(|V|+|E|)$ assembly types which also uses only a small constant number of fuel tiles for each transition, nine. An example of constructing an assembly set using this template follows in Section 4 where we build a base four oscillator. 


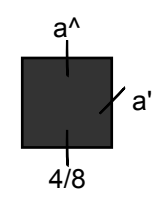

(a) This tile allows an edge gadget to detach the it's corresponding vertex.

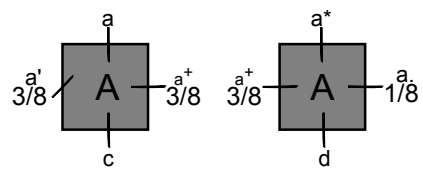

(b) These tiles together represent a vertex in the graph.

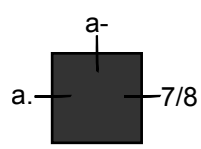

(c) This tile allows an edge gadget to know when it's vertex has finished attaching.

Figure 2: These four tiles are needed in order to represent any vertex in a graph. All glues labeled with some form of an $a$ require unique glues to the specific vertex these tiles represent and may not be shared amongst other vertices.

Vertex Representation. For each vertex in the graph you must have the set of two tiles shown in Figure $2 \mathrm{~b}$, a vertex detachment tile shown in Figure 2a, and finally a vertex attachment tile which is shown in Figure 2c. The five glues labeled $a^{\prime}, a^{*}, a ., a^{+}$and $a$ on the duple of Figure $2 \mathrm{~b}$ are unique to some vertex $a$ while the two southern glues are the same for all pairs of tiles.

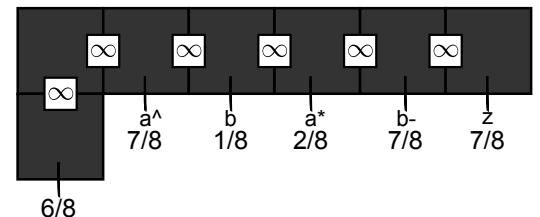

(a) This assembly, edge gadget, represent a directed edge from vertex $a$ to vertex $b$.

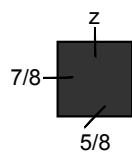

(b) This tile allows any edge gadget to detach itself once done.

Figure 3: The edge gadget and it's detachment tile.

Edge Gadget. Figure $3 \mathrm{a}$ is the edge gadget which exchanges a vertex $a$ with the vertex $b$ on the cell assembly. When the edge gadget detaches itself from the cell assembly all tiles that detach with the gadget are no longer usable. In Figure 3 a the glues labeled with some $a$ represent glues unique to some vertex $a$ while the glues labeled with some $b$ represent glues specific to some vertex $b$. The glue labeled with a $z$ is unique to the edge detachment tile shown in Figure 3b. This tile allows the edge gadget including it's junk to detach from the cell assembly.

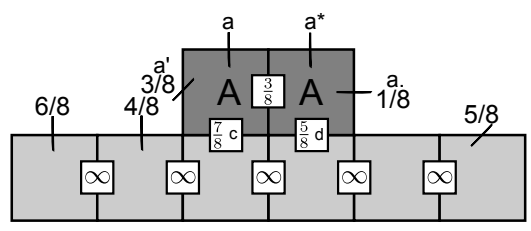

Figure 4: The initial pre-built starting cell assembly with a vertex already attached.

Cell Assembly. This cell assembly is assumed to be pre-built and contains the initial starting vertex $v$ already placed on top of the cell assembly. As you can see in Figure 4 the glues $c$ and $d$ are not discriminatory and allow any two tiles representing a vertex to attach to the cell assembly with the aid of an edge gadget. The $\frac{6}{8}^{t h}$ glue along with the $a *$ glue allow the edge gadget to attach. After the attachment the $\frac{4}{8}^{\text {th }}$ glue allows for the widget to get a better "grip" on the assembly cell so that it may detach the current vertex. The final $\frac{5}{8}^{\text {th }}$ glue on the assembly allows the edge gadget to pry itself off. 


\subsection{Correctness}

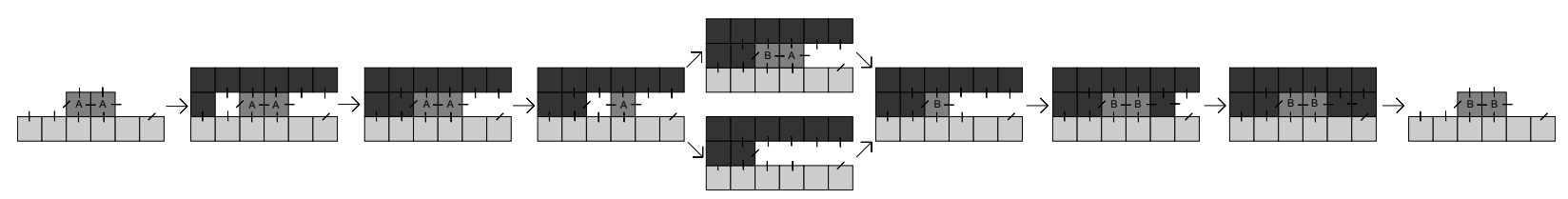

Figure 5: The steps necessary to transition from one state to another.

As can be seen from Figure 5 the possible transitions made by changing from one state to another are straight forward and verifying that each single tile addition must happen in the order shown is left as a short exercise to the reader. We will concentrate on the two negative glue detachment events which are more complex and need to be verified so as to ensure that no other cuts in the produced assemblies are unstable other than those depicted. We shall assume that each vertical column of the image represents a stage in the transition and so there are a total of 9 stages from start to finish.

\subsubsection{Correct Gadget Removal}

After the gadget is done a tile may attach to the negative $\frac{5}{8}^{\text {th }}$ glue on the cell assembly which in turn pops off the edge gadget along with any junk. Two scenarios that are brought about by the infinite binding within the cell assembly and edge gadget independently. The first scenario occurs when the cell assembly and edge gadget end up on opposite sides of the detachment. The other happens when both the cell assembly and edge gadget end up on the same side of the detachment. We will analyze both these scenarios and show that from stage 8 in Figure 5 the only valid detachment is what results in stage 9 .

Suppose both the cell and the gadget detach to opposite sides of the cut. We must include a negative glue to get an unstable cut and so the edge detachment tile must be on the same side as the gadget. This gives us a cut with weight $\frac{1}{8}$ not taking into account any of the other four labeled tiles. For the cut to be unstable we can only increase the weight by $\frac{6}{8}$ so as to keep it below 1 . To stay unstable both the vertex detachment tile and the vertex attached tile must be on the side of the edge gadget because of their $\frac{7}{8}$ attachment. Also, the left vertex tile must stay on the side of the cell assembly for the same reason. With this new information the total weight of the cut is now $\frac{6}{8}$ and we only have the right vertex tile to place. The right vertex tile must now stay on the side of the cell because to keep the cut unstable we may only increase the weight of the cut by $\frac{1}{8}$ more. Therefore, it is easy to see that the only possible unstable cut if both the cell and gadget are on opposite sides is the one displayed in Figure 5.

Now, suppose that both the cell assembly and the edge gadget detach to the same side of the cut. In order to include the negative strength of the edge detachment tile it must be on the opposite side of both the edge and the gadget. This immediately shows us that our cut is $\frac{9}{8}$ which is stable. With no other negative glue interactions, there is no cut with both the cell assembly and the edge gadget on the same side that would lead to a detachment.

Therefore, since we have exhaustively explored both scenarios using the fact that the cell assembly and edge gadget are infinitely bound we can say that the only unstable cut at stage 8 is the one that results in stage 9 .

\subsubsection{Correct Vertex Replacement}

Assume we are in stage 3 of Figure 5. We will show that the transition to stage 4 is the only valid detachment event that may happen. If the cell and edge gadget were to detach to opposite sides of the cut this would give us a cut with weight $\frac{5}{8}$. Then, no matter which side of the cut you place the vertex removal tile all cuts will be above 1. If both the edge gadget and the cell are on the same side of the cut, the left vertex tile must be on the opposite side of the cut since the vertex removal tile will not fall out. This means that either 
the left vertex tile or both vertex tiles must be on the opposite side of the cut. If both vertex tiles are on the opposite side of the cut we have a cut with weight $\frac{11}{8}$ which is stable. On the other hand, if it is only the left vertex tile on the opposite side of the cut then the weight of the cut is $\frac{7}{8}$ which enables it to detach. Therefore, the only detachment event that can occur after stage 3 is the single left vertex tile detaching from the assembly leaving the assembly in stage 4 . Once in stage 4 the right vertex tile destabilizes and may detach leaving room for the next vertex to attach and stage 6 to come about.

\section{Quaternary Oscillator}

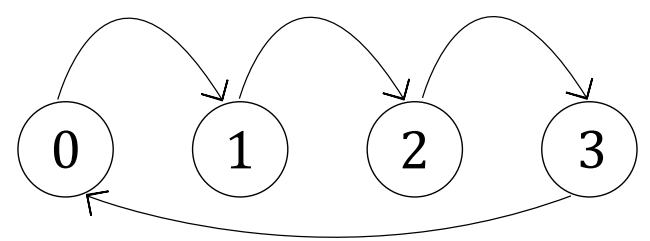

Figure 6: The directed graph of a quaternary oscillator.
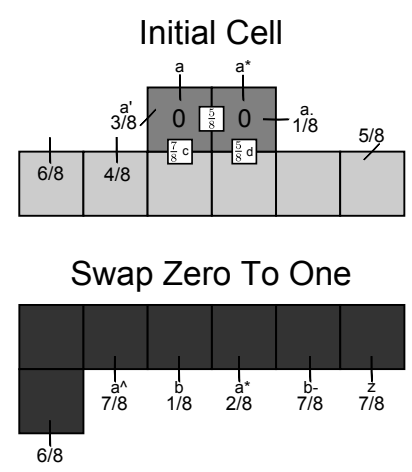

Swap One To Two

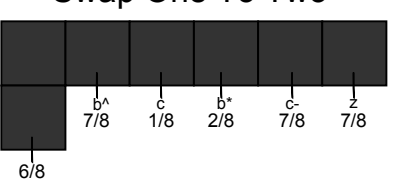

\section{Zero Vertex}
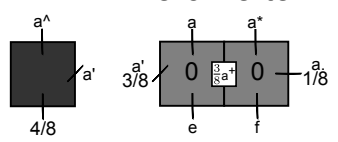

Two Vertex
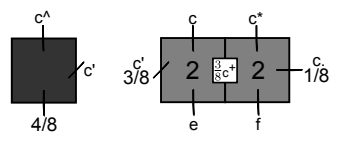

Swap Two To Three

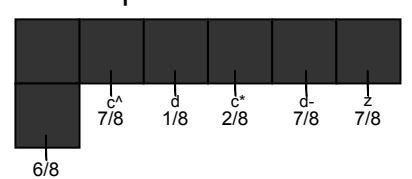

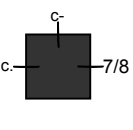
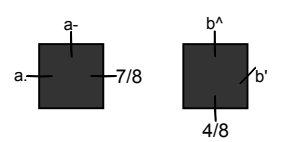

One Vertex

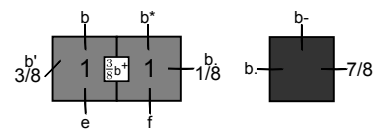

Three Vertex
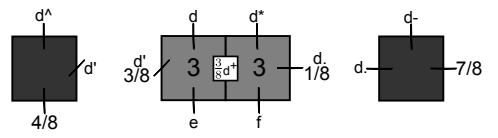

Figure 7: The complete assembly set for creating a quaternary oscillator.

A quaternary oscillator can be drawn as a directed graph with vertices representing each digit in the base-4 numeral system and the directed edges marking the transition from one numeral to the next. Figure 6 shows a directed graph representing a quaternary oscillator. As can be seen, every vertex has exactly one outgoing edge and as such even if our Graph Walking Template Assembly Set is non-deterministic there is only one valid walk through the graph making the assembly set deterministic. Figure 7 represents the complete assembly set necessary to represent the graph in Figure 6. This is a straightforward example of how to create a concrete assembly set using the assembly template presented in Section 3.1. 


\section{$5 \quad$ Fuel Efficient Universal Computation}

In this section we describe how to construct an initial assembly set $T$ that will simulate a given Turing machine $M$. To formally define simulation of a Turing machine, we utilize our graph walking formulation. For a given Turing machine $M$, we consider all possible tape configurations, states, and head locations the machine may enter over time and form a directed graph over these possible configurations depicting which configurations the machine may transition between within a single computation step. With this definition we say that an assembly set $T$ simulates $M$ if it walks this configuration graph.

Formally, we define the simulation as follows:

Turing Machine Simulation. Consider some Turing Machine $M=\left\langle Q, \Gamma, b, \Sigma, \delta, q_{0}, F\right\rangle$, an initial tape $t$, and head position $h$. Let a possible status of $M$ be a 3-tuple $S=\langle$ state, tape, head $\rangle$ where state represents some current state from $Q$, tape is some string of symbols over $\Gamma$, and head specifies a location on the tape where the Turing machine head currently sits. Additionally define the start status for a Turing machine $M$ and an input tape $r$ as the status corresponding to tape $=r$, head $=h$, and state $=q_{0}$.

Given a Turing machine $M$, define a directed graph $G_{M}$ where the set of all statuses for $M$ are vertices, and there exists an edge from any status $a$ to status $b$ if and only if $M$ can transition from status $a$ to $b$ in one step. Finally, we say an initial assembly set $T$ simulates Turing machine $M$ if $T$ walks $G_{M}$. Further, we say $T$ is a fuel-efficient simulation of $M$ if $T$ constitutes a fuel-efficient walk of $G_{M}$.

Our main result is as follows:

Theorem 5.1. For any Turing machine $M$, there exists an initial assembly set $T$ such that $T$ is a fuel-efficient simulation of $M$. Further, $|T|=O(|\Gamma| \cdot|Q|)$, and each element of $T$ is of $O(1)$ size.

As with our graph walking theorem, it is possible to strengthen this theorem such that $T$ contains only singleton tile assemblies. For clarity of presentation and space, we omit the extended construction that achieves this.

The remainder of this section presents the construction that proves Theorem 5.1

\subsection{Template Tile Set}
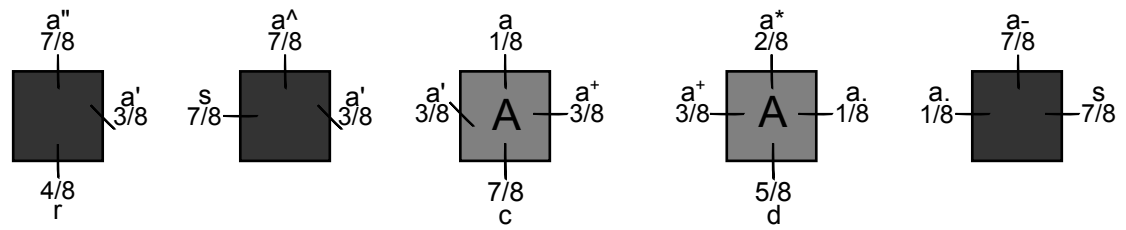

Figure 8: These tiles represent a symbol and state pair along with their needed utility tiles.

Symbols And States. For our construction we add an additional state, the empty state $\varnothing$, to $M$ which will be used to represent a symbol with no state i.e. a location where the head of the Turing Machine currently is not. For every symbol in $\Gamma$ and every state in $Q$ including $\varnothing$ we create the set of tiles shown in Figure 8. This means that we should have exactly $|\Gamma| \cdot(|Q|+1)$ sets of tiles. The two center tiles labeled with an $A$ in Figure 8 will represent some duple $(a \in Q, s \in \Gamma \cup \varnothing)$. We define a stateless state symbol pair as having the $\varnothing$ state while a stateful state symbol pair has some $q \in Q$. The two leftmost tiles are called state symbol detachment tiles and detach the particular state symbol tile pair they were created with. This will be useful later when trying to transition the state of the Turing Machine. Finally, the last tile on the right is the state symbol attached tile and will allow the assemblies to know that the state symbol tile pair associated with it have both been attached. 

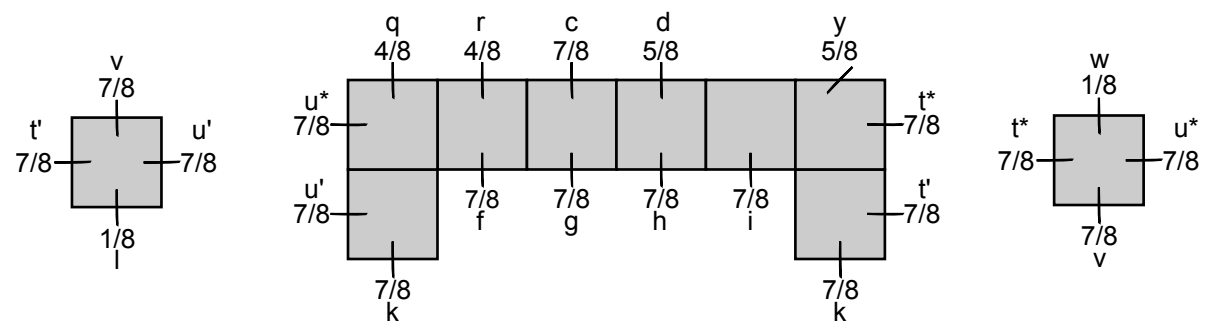

Figure 9: This assembly represents a single position on the tape. It is a tape cell which can and should contain some symbol state pair atop it attached to glues $c$ and $d$. The two accompanying tiles are what will allow the tape to extend to the right or left.

Tape Cell. A tape cell in our construction is a single piece of tape which contains some state symbol tile pair taken from Figure 8. If the state contained on the tape cell is $\varnothing$ then the tape cell contains only data, i.e. a symbol from $\Gamma$. On the other hand, if the state contained on the tape is not $\varnothing$ then the location of the Turing Machine's head is at that location and it contains some symbol from $\Gamma$. The whole of the tape is then at least one tape cell possibly combined with many more tape cells attached together with the head of the tape in only a single location i.e. with a single cell containing a state unequal to $\varnothing$ at any given time. Should the head of the Turing Machine be placed in multiple locations it will result in undefined behavior. When we later extend the tape we insure that the extended piece of tape gets initialized automatically to the blank state; i.e. the symbol $b$ in combination with the state $\varnothing$.
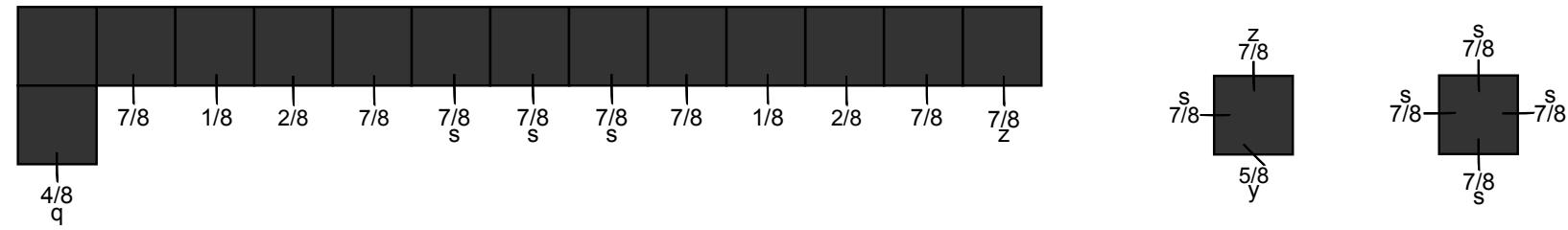

Figure 10: The leftmost assembly represents a single "Turing Instruction" and is called a transition gadget. The second tile is the detachment tile and the last tile is simply a tile that will be used to fill space.

Transition Function. A Turing Instruction is the 5-tuple $I=\langle c \in Q, s \in \Gamma, p \in \Gamma,\{L, R\}, n \in Q\rangle$ where $c$ is the current state, $s$ is the scanned symbol, $p$ is the symbol to print, $\{L, R\}$ is the left or right movement of the head, and $n$ is the new state. For each Turing Instruction in $M$ create $|\Gamma|$ transition gadgets. A single transition gadget is shown in Figure 10.

For two state symbol pairs we follow the same process as exchanging a single vertex in the Graph Walking Template with a few exceptions. The most obvious exception is that we are now exchanging two symbol state pairs instead of a single vertex pair. In order to facilitate this change we extend the transition gadget to accompany the two exchanges. The next difference is that after exchanging the first pair of tiles the next symbol state pair must be exchanged instead of detaching the whole transition gadget. This is the reason for needing two state symbol detachment tiles instead of just the single one as in the Graph Walking Template. Now, of the two detachment tiles the first is used when detaching the left state symbol pair underneath the transition gadget and the second is used when detaching the right state symbol pair underneath the transition gadget. This along with the changing of a couple glue strengths gives us a transition gadget shown in Figure 10 that is able to exchange two symbol state pairs at the same time.

Now in order to create the transition gadgets which represents a Turing instruction we must properly set the label of several unlabeled glues. We will temporarily number the unlabeled glues in Figure 10 from 1 starting at the left to 8 at the farthest right. It does not matter whether you are moving the head left or 

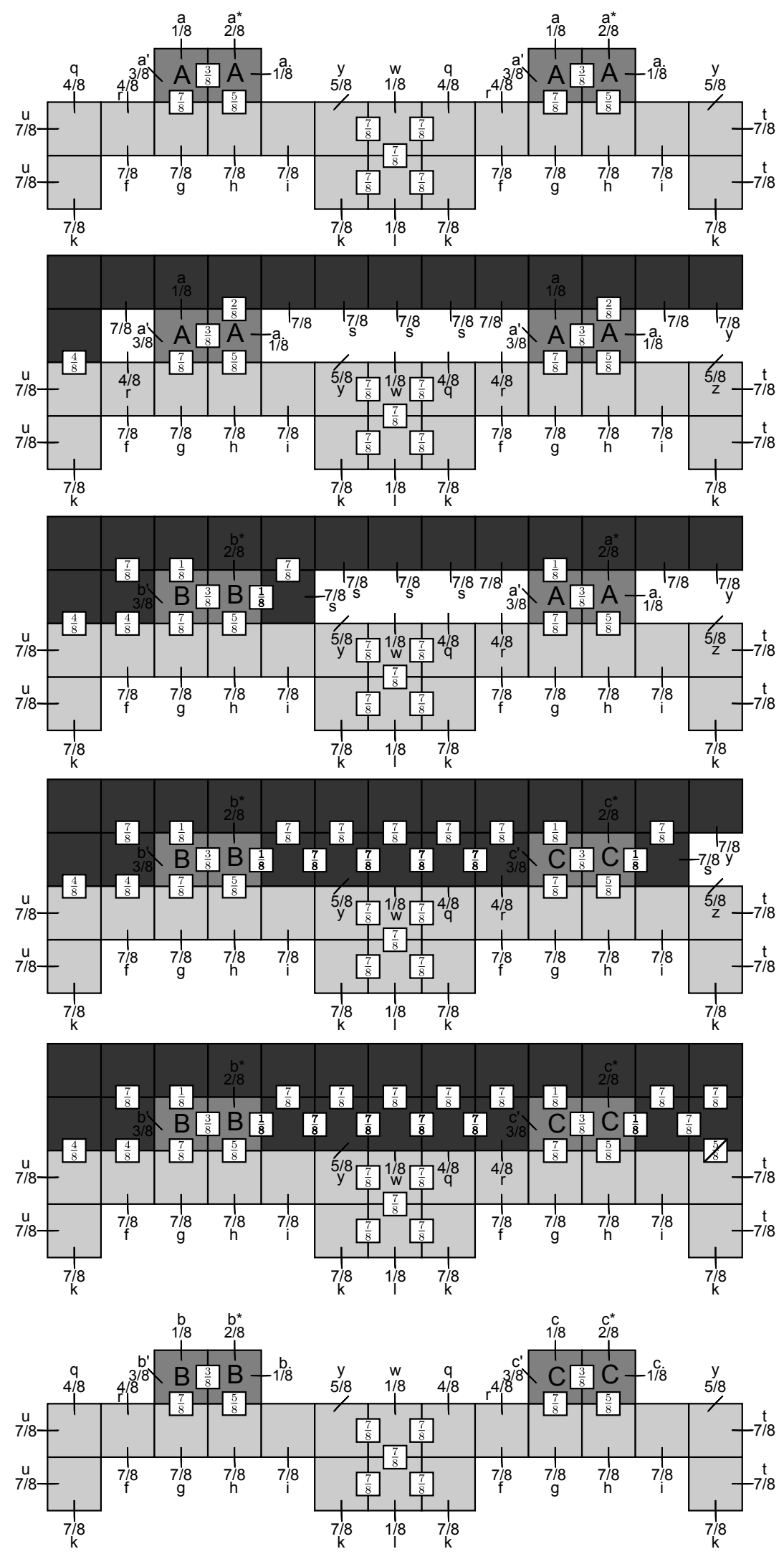

Figure 11: This is an example of a transition gadget changing two As into an B and a C. 
right, the stateful pair always becomes stateless and the stateless pair always becomes stateful. Therefore, the only difference between moving the head right or left is that the stateful pair is at the left when moving right or at the right when moving left. We will continue assuming we are creating the transition gadgets for some $I$ which moves the head to the right. Since we are moving right, glues 3 and 1 will use the proper glue labels for the state symbol pair $(c, s)$. Glues 2 and 4 use the proper glue labels from the state symbol pairs $(\varnothing, p)$. Now, the next part explains the reason why we need $|\Gamma|$ gadgets. All gadgets now have glues 1 through 4 filled in with labels from our assembly set. Next fill in glues 5 and 7 for all gadgets with the proper glue labels from every state symbol pair that contains the $\varnothing$ state. Finally glues 6 and 8 are filled using the glue labels for every state symbol pair that contains state $n$ such that the symbol represented in 5 and 7 matches those of 6 and 8 within the same transition gadget. Figure 11 contains an overview of the necessary steps that allow the transition transition gadget to work.

With the gadget just about to detach, we can use the same arguments presented in Section 3.2 to prove that the transition gadget detaches correctly. The tape cell and transition gadget themselves are strongly connected and should not fall apart. Therefore, we start by saying that either both the tape cell and transition gadget end up on the same side of the cut or they end up on different sides of the cut. Using this information and the fact that we want a cut below 1 we can whittle down the possible sides of all the tiles in either situation. As one will be able to see the only possible cut below 1 happens when the tape cell and transition gadget are on opposite sides of the cut and all the fuel tiles are on the same side as the transition gadget.
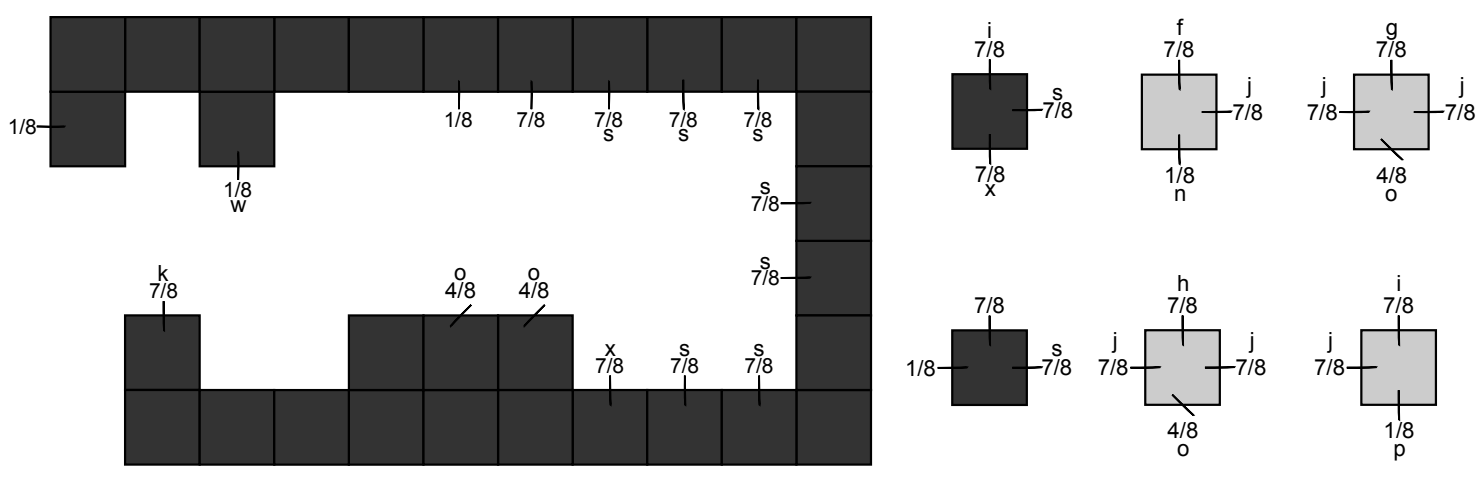

Figure 12: The assembly needed to extend the tape to the right including two utility tiles colored dark gray. The four light gray tiles are necessary to allow the tape extension gadgets to remove themselves and not continuously attach. For extending to the left see Figure 13 which is in the Appendix.

Tape Extension. In order to simulate a Turing Machine and be space efficient we must ensure that our tape does not grow infinitely but extends only when needed. Our construction ensures that our tape grows only when necessary. We use a recurring theme that has been presented throughout the paper so far which is to place what we need and then use some negative glues to destabilize the then junk assembly. Due to some symmetric differences tape extension to the left is a bit different from that of the right but both work identically. The similarity between the two extension gadgets allows us to simply examine a single one and so only the right extension gadget will be discussed.

We will need four tiles, shown in Figure 12, which will serve as a way for the tape extension gadgets to detach themselves once they are done with their work. For simplicity we create a tape extension gadget for every stateful state symbol pair. Since it is only a single tile that needs to change in every tape extension gadget we could separate that single tile and just create a single tile for every stateful state symbol pair. The tape extension gadget can only attach if the position of the head is at the edge of the tape i.e. the stateful state symbol pair is at the edge. Once the head makes its way to the edge of the tape the tape extension gadget is able to attach and extend the tape. Then the tape extension gadget places the two tiles 


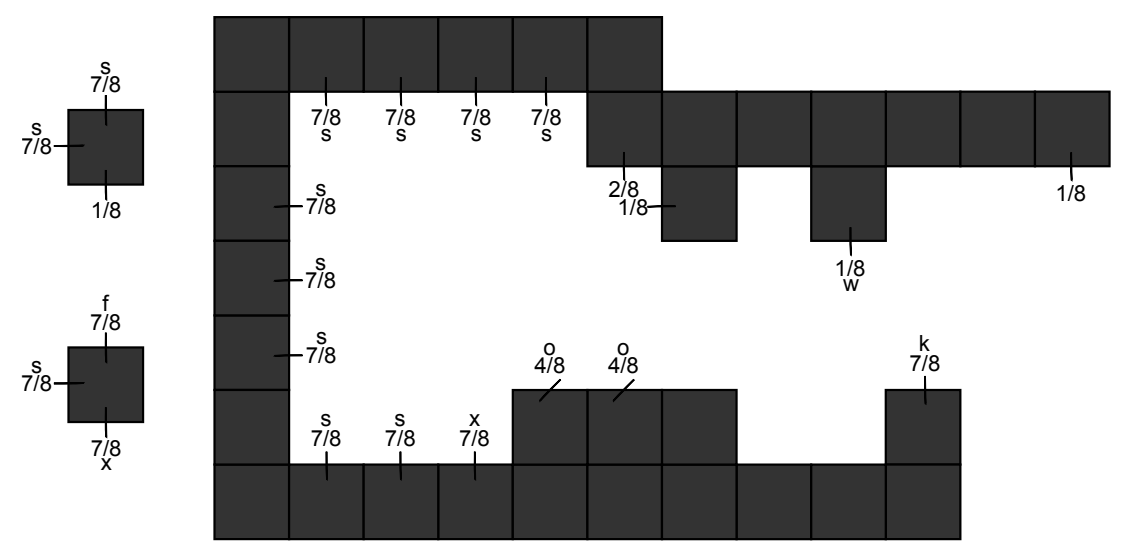

Figure 13: The assembly needed to extend the tape to the left including two utility tiles.

shown in Figure 9 which will allow another tape cell assembly to attach. Once the new tape cell assembly has attached the stateless blank or "default" state symbol pair can then be attached to the tape cell. After the attachment of the state symbol pair, tiles cooperatively bind around the tape extension arch until it attaches a tile underneath the tape cell arch. Finally, three tiles attach which cause the detachment of the extension gadget. A detailed overview of this process can be seen in Figure 14. The proof that the tape extension gadget detaches properly is easily verifiable at this point and is left as an exercise to the reader.

\subsection{Tape Reduction}

A Turing machine in theory has an infinite length tape to work with. To simulate this property our construction automatically extends the finite length tape on demand when needed. However, it is reasonable that a Turing machine may grow a large tape, and then subsequently "blank" out much of the tape leaving only a small length portion of tape between the leftmost and rightmost non-blank symbol. In some sense, it is a waste of space to explicitly encode the large number of blank bits that are beyond this region.

To not waste this space requires the inclusion of an additional mechanism into our construction, the tape reduction gadget. This gadget simply clips off the left or rightmost bit of the tape under certain circumstances such as if the leftmost or rightmost bit has been blanked. This gadget allows for our Turing machine to achieve space efficiency, which is the requirement that the size of the assembly representing each computational step is asymptotically bounded above by the size of the region of the tape between the leftmost and rightmost non-blank symbol, or the region between the head position and either the right or leftmost non-blank symbol.

One motivation for achieving space-efficiency is the potential implementation of components from active self-assembly models within our passive tile assembly model. In particular, our space efficient construction may be utilized as the internal workings of a larger active component. While the computation required of an active component may require a large tape size, the final output may be taken from a small set of options such as "turn on glue a", or something similar. With space efficiency, there is hope that our passive implementation will be able to perform the required functionality without growing so large that the geometry of the mechanism interferes with the higher-level active system mechanics.

Figure 15 shows the gadget that is necessary to shrink the tape from the right. One end of this gadget connects to a "blank" bit while the other end connects to a bit and state combination that will then continue to blank the bit the head is at. An example of tape reduction can be seen in Figure 17 which shows an abridged set of steps necessary to make the tape shrink on the left end. Without any way to synchronize the shrinking of the tape with the movement of the head one may still end up with many sequential "blank" bits at the end of the tape. In order to prevent this, whenever a transition gadget wants to "blank" a bit on some end of the tape one must add a tail, such as the one shown in Figure 16, to the transition gadget. 

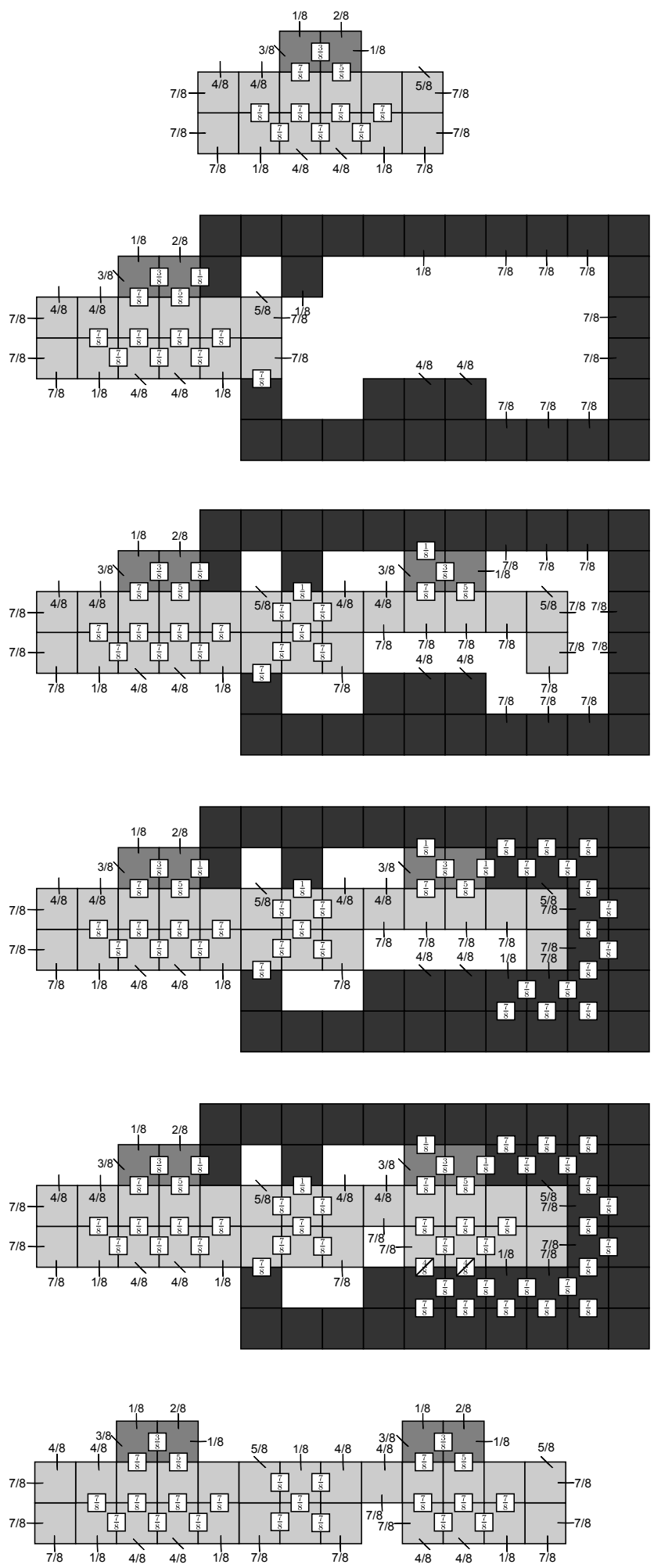

Figure 14: This figure shows the steps that occur when extending the tape to the right. 

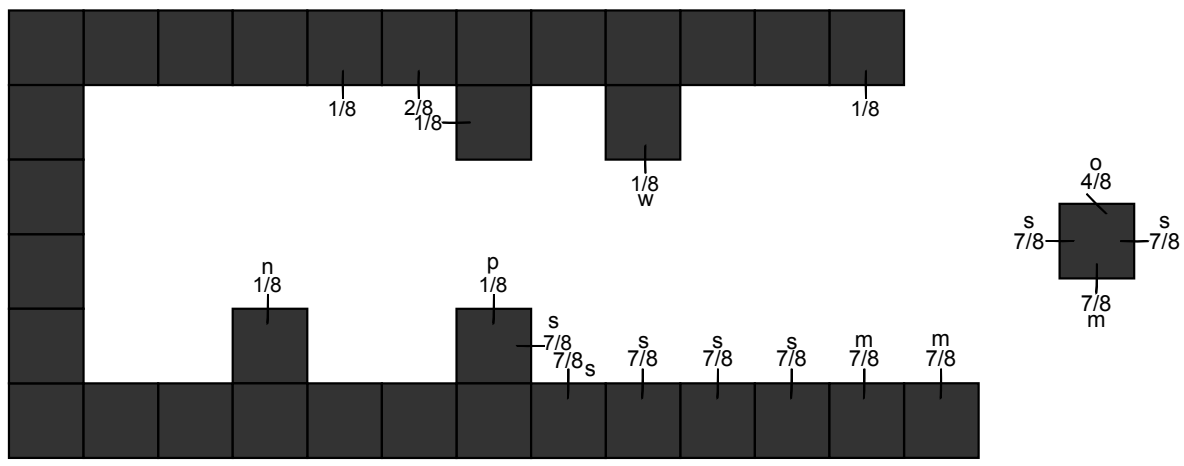

Figure 15: The tape reduction gadget and extra tile needed to remove a single tape cell from the left edge. This gadget relies on other tiles that have been presented in this paper.

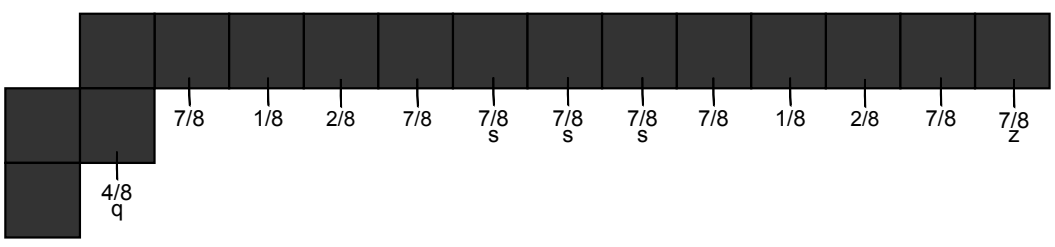

Figure 16: This is an example of how a tail would be placed on a transition gadget that will "blank" the left bit and move right.
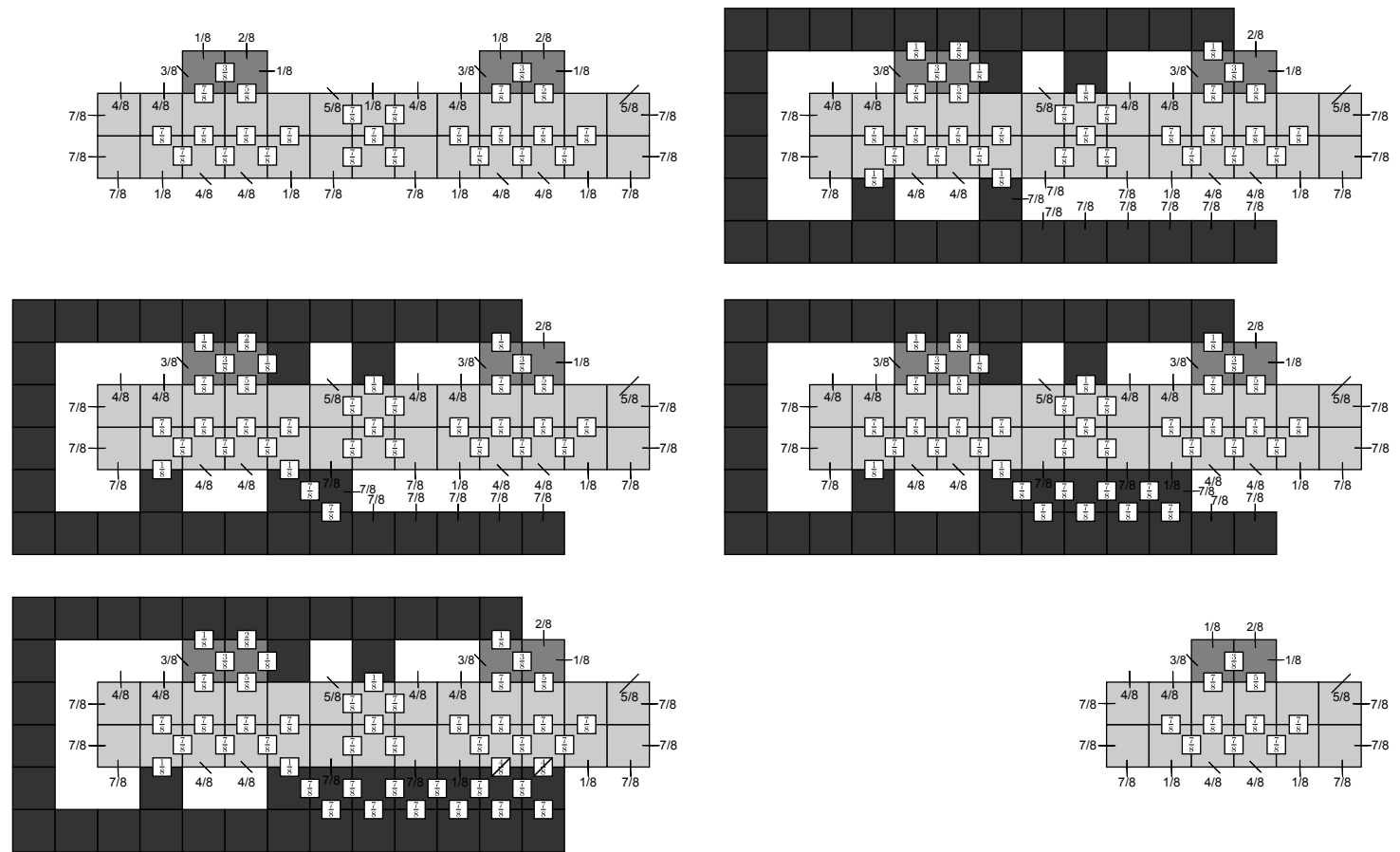

Figure 17: This shows how a piece of tape will use the tape reduction gadget to shrink by one cell at the left end. 
This tail must be added on the side that will be "blanked" so as to be sure that all blank bits have been removed by tape reduction gadgets.

\section{References}

[1] Zachary Abel, Nadia Benbernou, Mirela Damian, Erik Demaine, Martin Demaine, Robin Flatland, Scott Kominers, and Robert Schweller, Shape replication through self-assembly and RNase enzymes, SODA 2010: Proceedings of the Twenty-first Annual ACM-SIAM Symposium on Discrete Algorithms (Austin, Texas), Society for Industrial and Applied Mathematics, 2010.

[2] Leonard Adleman, Qi Cheng, Ashish Goel, and Ming-Deh Huang, Running time and program size for self-assembled squares, Proceedings of the thirty-third annual ACM Symposium on Theory of Computing (New York, NY, USA), ACM, 2001, pp. 740-748.

[3] Leonard Adleman, Qi Cheng, Ashish Goel, Ming-Deh Huang, and Hal Wasserman, Linear selfassemblies: Equilibria, entropy and convergence rates, In Sixth International Conference on Difference Equations and Applications, Taylor and Francis, 2001.

[4] Leonard M. Adleman, Qi Cheng, Ashish Goel, Ming-Deh A. Huang, David Kempe, Pablo Moisset de Espanés, and Paul W. K. Rothemund, Combinatorial optimization problems in self-assembly, Proceedings of the Thirty-Fourth Annual ACM Symposium on Theory of Computing, 2002, pp. 23-32.

[5] Leonard M. Adleman, Jarkko Kari, Lila Kari, Dustin Reishus, and Petr Sosík, The undecidability of the infinite ribbon problem: Implications for computing by self-assembly, SIAM Journal on Computing $\mathbf{3 8}$ (2009), no. 6, 2356-2381.

[6] Gagan Aggarwal, Qi Cheng, Michael H. Goldwasser, Ming-Yang Kao, Pablo Moisset de Espanés, and Robert T. Schweller, Complexities for generalized models of self-assembly, SIAM Journal on Computing 34 (2005), 1493-1515, Preliminary version appeared in SODA 2004.

[7] Robert D. Barish, Rebecca Schulman, Paul W. Rothemund, and Erik Winfree, An information-bearing seed for nucleating algorithmic self-assembly, Proceedings of the National Academy of Sciences 106 (2009), no. 15, 6054-6059.

[8] Florent Becker, Ivan Rapaport, and Eric Rémila, Self-assembling classes of shapes with a minimum number of tiles, and in optimal time, Foundations of Software Technology and Theoretical Computer Science (FSTTCS), 2006, pp. 45-56.

[9] Nathaniel Bryans, Ehsan Chiniforooshan, David Doty, Lila Kari, and Shinnosuke Seki, The power of nondeterminism in self-assembly, SODA 2011: Proceedings of the 22nd Annual ACM-SIAM Symposium on Discrete Algorithms, SIAM, 2011, pp. 590-602.

[10] Sarah Cannon, Erik D. Demaine, Martin L. Demaine, Sarah Eisenstat, Matthew J. Patitz, Robert Schweller, Scott M. Summers, and Andrew Winslow, Two hands are better than one (up to constant factors), Arxiv preprint arXiv:1201.1650 (2012).

[11] Harish Chandran, Nikhil Gopalkrishnan, and John H. Reif, The tile complexity of linear assemblies, 36th International Colloquium on Automata, Languages and Programming, vol. 5555, 2009.

[12] Ho-Lin Chen and David Doty, Parallelism and time in hierarchical self-assembly, SODA 2012: Proceedings of the 23rd Annual ACM-SIAM Symposium on Discrete Algorithms, SIAM, 2012.

[13] Ho-Lin Chen, David Doty, and Shinnosuke Seki, Program size and temperature in self-assembly, ISAAC 2011: Proceedings of the 22nd International Symposium on Algorithms and Computation, Lecture Notes in Computer Science, vol. 7074, Springer-Verlag, 2011, pp. 445-453. 
[14] Matthew Cook, Yunhui Fu, and Robert T. Schweller, Temperature 1 self-assembly: Deterministic assembly in $3 d$ and probabilistic assembly in 2d, SODA, 2011, pp. 570-589.

[15] Erik D. Demaine, Martin L. Demaine, Sándor P. Fekete, Mashhood Ishaque, Eynat Rafalin, Robert T. Schweller, and Diane L. Souvaine, Staged self-assembly: nanomanufacture of arbitrary shapes with O(1) glues, Natural Computing 7 (2008), no. 3, 347-370.

[16] Erik D. Demaine, Sarah Eisenstat, Mashhood Ishaque, and Andrew Winslow, One-dimensional staged self-assembly, Proceedings of the 17th international conference on DNA computing and molecular programming, DNA'11, 2011, pp. 100-114.

[17] Erik D. Demaine, Matthew J. Patitz, Robert T. Schweller, and Scott M. Summers, Self-assembly of arbitrary shapes using RNase enzymes: Meeting the Kolmogorov bound with small scale factor, STACS 2011: Proceedings of the 28th International Symposium on Theoretical Aspects of Computer Science, 2011.

[18] David Doty, Randomized self-assembly for exact shapes, SIAM Journal on Computing, to appear.

[19] David Doty, Lila Kari, and Benoît Masson, Negative interactions in irreversible self-assembly, DNA 16: Proceedings of The Sixteenth International Meeting on DNA Computing and Molecular Programming, Lecture Notes in Computer Science, Springer, 2010, pp. 37-48.

[20] David Doty, Jack H. Lutz, Matthew J. Patitz, Robert Schweller, Scott M. Summers, and Damien Woods, The tile assembly model is intrinsically universal, FOCS 2012: Proceedings of the 53rd IEEE Conference on Foundations of Computer Science, 2012.

[21] David Doty, Matthew J. Patitz, Dustin Reishus, Robert T. Schweller, and Scott M. Summers, Strong fault-tolerance for self-assembly with fuzzy temperature, FOCS 2010: Proceedings of the 51st Annual IEEE Symposium on Foundations of Computer Science, IEEE, 2010, pp. 417-426.

[22] Bin Fu, Matthew J. Patitz, Robert Schweller, and Robert Sheline, Self-assembly with geometric tiles, ICALP 2012: Proceedings of the 39th International Colloquium on Automata, Languages and Programming (Warwick, UK), 2012.

[23] Kenichi Fujibayashi, Rizal Hariadi, Sung Ha Park, Erik Winfree, and Satoshi Murata, Toward reliable algorithmic self-assembly of dna tiles: A fixed-width cellular automaton pattern nano letters, (2007).

[24] Ming-Yang Kao and Robert T. Schweller, Reducing tile complexity for self-assembly through temperature programming, SODA 2006: Proceedings of the 17th Annual ACM-SIAM Symposium on Discrete Algorithms, 2006, pp. 571-580.

[25] _ Randomized self-assembly for approximate shapes, International Colloqium on Automata, Languages, and Programming, Lecture Notes in Computer Science, vol. 5125, Springer, 2008, pp. 370-384.

[26] Ján Maňuch, Ladislav Stacho, and Christine Stoll, Step-assembly with a constant number of tile types, ISAAC 2009: Proceedings of the 20th International Symposium on Algorithms and Computation (Berlin, Heidelberg), Springer-Verlag, 2009, pp. 954-963.

[27] Jennifer E. Padilla, Matthew J. Patitz, Raul Pena, Robert T. Schweller, Nadrian C. Seeman, Robert Sheline, Scott M. Summers, and Xingsi Zhong, Asynchronous signal passing for tile self-assembly: Fuel efficient computation and efficient assembly of shapes, Arxiv preprint arXiv:1202.5012 (2012).

[28] Matthew J. Patitz, Robert T. Schweller, and Scott M. Summers, Exact shapes and turing universality at temperature 1 with a single negative glue, Proceedings of the Seventeenth International Conference on DNA Computing and Molecular Programming (DNA 17) (California Institute of Technology, Pasadena, CA), 2011. 
[29] Paul W.K. Rothemund, Nick Papadakis, and Erik Winfree, Algorithmic self-assembly of DNA Sierpinski triangles, PLoS Biology 2 (2004), no. 12, 2041-2053.

[30] David Soloveichik and Erik Winfree, Complexity of self-assembled shapes, SIAM Journal on Computing 36 (2007), no. 6, 1544-1569.

[31] Scott M. Summers, Reducing tile complexity for the self-assembly of scaled shapes through temperature programming, Algorithmica 63 (2012), no. 1, 117-136.

[32] Erik Winfree, Algorithmic self-assembly of DNA, Ph.D. thesis, California Institute of Technology, June 1998.

[33] Damien Woods, Ho-Lin Chen, Scott Goodfriend, Nadine Dabby, Erik Winfree, and Peng Yin, Efficient active self-assembly of shapes, Manuscript (2012). 